\title{
Desenvolvimento de um Assistente Virtual Integrado ao Moodle para Suporte a Aprendizagem Online
}

\author{
Alexandre M. A. Maciel $^{1}$, Rodrigo L. Rodrigues ${ }^{2}$, Edson C. B. Carvalho ${ }^{3}$ \\ ${ }^{1}$ Escola Politécnica - Universidade de Pernambuco (UPE) \\ Rua Benfica, 415 - Madalena - CEP: 50720-001 - Recife/PE \\ 2 Departamento de Educação - Universidade Federal Rural de Pernambuco (UFRPE) \\ Rua Dom Manoel de Medeiros, s/n, Dois Irmãos - CEP: 52171-900 - Recife/PE \\ ${ }^{3}$ Centro de Informática - Universidade Federal de Pernambuco (UFPE) \\ Av. Jornalista Aníbal Fernandes, s/n - Cidade Universitária - 50.740-560 - Recife/PE \\ amam@ecomp.poli.br, rlr@ded.ufrpe.br, ecdbcfecin.ufpe.br
}

\begin{abstract}
The use of animated virtual assistants in learning environments has become an increasingly important feature of affectivity in order to promote interactivity in teaching relation. This paper describes the design, development and evaluation of a plugin for Moodle called Avatar Education proposed for everyday support for students in Distance Learning courses. Experiments were performed in a real teaching environment and the results reached levels expected user satisfaction.
\end{abstract}

Resumo. $O$ uso de assistentes virtuais animados em ambientes de aprendizagem tem se tornado cada vez mais um recurso de afetividade importante no intuito de promover interatividade na relação de ensino. Este artigo descreve a concepção, desenvolvimento e avaliação de um plugin para Moodle chamado Avatar Educação proposto para o suporte a aprendizagem online. Experimentos foram realizados em um ambiente real de ensino e os resultados atingiram os níveis de satisfação do usuário esperados.

\section{Introdução}

Nos últimos anos, tornou-se notável o interesse pelo estabelecimento de relações sociais entre humanos e interfaces virtuais, e isto tem despertado o interesse da comunidade científica, que cada vez mais propõe novos sistemas especializados neste tipo de interação humano-computador (Duarte e Costa, 2005).

No âmbito educacional, diferentes ferramentas têm sido propostas como forma de oferecer meios de integração de recursos textuais, de som e de imagem, além de apoio para as interações entre professores e estudantes (Lyra e Santos, 2012). Essas ferramentas cada vez mais incorporam novas abordagens de comunicação e colaboração e oferecem recursos importantes para o desenvolvimento da aprendizagem interativa.

Diante desse cenário, é crescente o uso de assistentes virtuais de aprendizagem, que interagem com o estudante em ambientes de aprendizagem. Segundo Prola e Viccari (2003) esses personagens aumentam a afetividade das aplicações de educação e podem ser empregados em muitas aplicações interativas e que auxiliem estudantes. 
Dentre as inúmeras aplicações que podem receber a incorporação de assistentes virtuais estão os Ambientes Virtuais de Aprendizado (AVA), que cada vez mais têm ganhado grande destaque na educação acadêmica e corporativa. Os AVA normalmente fornecem um conjunto de ferramentas tais como: chat, fórum de discussão, postagem de materiais, web conferência, viabilizando a comunicação síncrona e assíncrona entre professores, tutores e alunos envolvidos nos cursos de Ensino a Distância (EAD).

Considerando o Moodle, como um dos AVA's mais utilizados no Brasil, observa-se que entre os plug-ins oficiais não há disponibilidade de personagens virtuais com objetivo de fornecer suporte à aprendizagem. Associado a isto, diversas críticas dos alunos vêm sendo relatadas em relação à quantidade de informações diárias, de prazos e de recursos, atribuídos constantemente no decorrer da disciplina. Desse modo, o acompanhamento do fluxo de informação torna-se bastante complexo.

Em Rodrigues et al. (2012) foi desenvolvido uma ferramenta para produção de vídeo aulas utilizando personagem animado com síntese de voz. Nesse trabalho, foi construída uma arquitetura de desenvolvimento portável por meio de um middleware chamado Avatar Vocálico responsável pela geração das sequências de animação e por realizar a comunicação com o Framework FIVE (Maciel e Carvalho, 2010) para a geração do áudio sintético. A partir desse arcabouço, o desenvolvimento automatizado desse tipo de animação tornou-se mais rápido e barato, sendo possível a extensão do mesmo para outros ambientes tecnológicos.

Tendo em vista essa característica de portabilidade de plataforma, este artigo objetiva apresenta a concepção, desenvolvimento e avaliação de um assistente virtual para o suporte a aprendizagem online, integrado ao AVA Moodle, chamado Avatar Educação. Desenvolvido em formato de plugin, este recurso faz uso do middleware Avatar Vocálico desenvolvido em Rodrigues et al. (2012) e do Framework FIVE (Maciel e Carvalho, 2010). Este trabalho foi realizado em cooperação com a empresa Vocal Lab e financiado pelo Programa SEBRAEtec Inova do SEBRAE.

As próximas seções do artigo estão organizadas da seguinte maneira: a seção dois apresenta a revisão da literatura referente ao foco deste trabalho, abordando as tecnologias utilizadas e os conceitos relacionados, a seção três apresenta o processo de concepção e desenvolvimento do assistente virtual e sua arquitetura de funcionamento, a seção quatro apresenta os experimentos, resultados e discussão realizados para validação da ferramenta, e por último a seção cinco apresenta as considerações finais e as possibilidades de trabalhos futuros.

\section{Revisão da Literatura}

Esta revisão da literatura apresenta os conceitos essenciais para o entendimento do problema de pesquisa e para o desenvolvimento do assistente virtual. Dentre os conceitos apresentados, analisamos o uso de assistentes virtuais em ambientes de aprendizagem, a apresentação da técnica de animação baseada em visemas, e por fim, o processo de síntese de fala.

\subsection{Uso de Assistentes Virtuais em Ambientes de Aprendizagem}

No tocante aos aspectos tecnológicos, nos últimos anos tem-se empregado cada vez mais softwares como um recurso de suporte educacional a gestores, professores e 
estudantes. Além disso, a mudança do foco no processo educacional promoveu a evolução significativa destes recursos, que passaram a se preocupar mais com a aprendizagem do que com o ensino (Rissoli, 2010).

O uso de assistentes virtuais em ambientes de aprendizagem é uma ideia que remota a década de 1990. Trabalhos como o de Lester et al.(1997) mostram que agentes animados oferecem uma grande promessa para a entrega em tempo real de recomendações para resolução de problemas sofisticados, com forte apelo visual. Adicionalmente, a possibilidade de aumentar a efetividade do aprendizado dos estudantes com feedbacks customizados, por meio de agentes pedagógicos animados, pode oferecer outro importante benefício: motivação.

Com base na promoção e aumento do uso destas tecnologias, a pesquisa e desenvolvimento na área de assistentes virtuais de aprendizagem vem avançando fortemente. Cada vez mais, este tipo de recurso tem procurado adequar as estratégias de ensino às necessidades de aprendizagem dos usuários, fornecendo-lhes apoio educacional durante seu processo de ensino-aprendizagem.

Normalmente a construção destes personagens em softwares educacionais é feita com ferramentas que exigem um conhecimento específico de artes, modelagem e animação. O processo de inserção da voz geralmente é feito de forma artesanal, ou seja, são criadas animações em diferentes contextos de interação, e posteriormente é gravado a voz de um locutor que ao final é inserida na animação desenvolvida. Esse processo, na maioria das vezes, demanda de um alto investimento e alta carga de trabalho, pois necessita de locutores, estúdios de gravação de voz, artistas, animadores e roteiristas Rodrigues et al. (2012). Existem diversas formas para construção das animações utilizadas nesses ambientes, um das mais utilizadas são as baseadas em visemas.

\subsection{Animação Baseada em Visemas}

Segundo Gratch (2002), os personagens virtuais animados são recursos audiovisuais capazes de capturar facilmente a atenção do usuário e tornar a atividade de interação mais atrativa e envolvente. Para que isso ocorra de modo efetivo é fundamental que seja realizado um grande esforço multidisciplinar. Conhecimentos em áreas como processamento de voz, processamento da linguagem natural, inteligência artificial, computação gráfica e animação são fundamentais para o desenvolvimento de personagens cada vez mais próximos da realidade.

Dependendo da aplicação dada aos personagens virtuais animados, eles podem desempenhar papéis variados, tais como instrutores, assistentes, apresentadores, atendentes ou vendedores. Especificamente na área de educação esses personagens podem ser usados como agentes pedagógicos com o objetivo de aumentar a efetividade das aplicações de educação e treinamento, ou podem também ser empregados em aplicações interativas e que auxiliem usuários (Prola, 2003).

A presença deste tipo de personagem em aplicações de computadores normalmente pode ser utilizada de corpo inteiro ou apenas por meio de "cabeças falantes", considerando o papel de destaque que a face ocupa na comunicação humana. Os mecanismos de comunicação face a face, estimulados pelas experiências sociais, nos tornam capazes de interpretar e identificar estados emocionais transmitidos pela face 
utilizando sua informação visual para complementar a compreensão da mensagem transportada pelo sinal acústico da fala.

Uma maneira bastante trivial de se conseguir modelar e animar faces em um grau de realismo simples porém satisfatório é por meio da utilização de visemas. Segundo Costa (2009), visemas são imagens de posturas labiais visualmente contrastantes entre si, associadas aos diversos sons da fala (fonemas). O processo de animação baseada em visemas consiste em duas fases: a fase de construção de uma base de imagens com a reprodução realista da movimentação articulatória visível da fala e dos efeitos da coarticulação; e a fase de síntese em sistemas de animação facial na qual é gerada uma animação em sincronia e harmonia com o áudio correspondente à fala. A Figura 1 mostra um esquema geral deste processo.

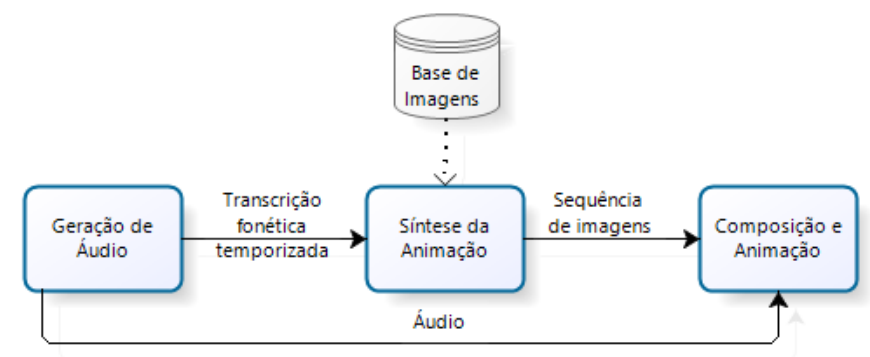

Figura 1. Síntese de Animação Facial 2D [26].

O processo de síntese de animação facial inicia com a definição do áudio correspondente à fala, que pode ser resultado de um processo de gravação da voz de um locutor ou de um sistema de síntese da fala. A partir do áudio gerado obtém-se a transcrição fonética temporizada da fala a ser animada. Essa transcrição é composta pela sequência de fonemas que compõem a locução e suas respectivas durações. As informações fornecidas pela transcrição fonética temporizada são então utilizadas pelo sistema para selecionar os visemas correspondentes aos fonemas transcritos da base de imagens e, após processá-las, gerar uma sequência de quadros que reproduzem na face virtual a movimentação articulatória visível. Por fim, a composição e apresentação da animação, que pode ser construída por meio de diversas técnicas de animação tais como: metamorfose, síntese concatenativa e trajetória de visemas (Costa, 2009).

\subsection{Síntese de Fala}

Nas últimas décadas, a área de interface de voz tem recebido grande atenção da academia, em virtude de dois fatores: primeiro, devido à melhoria no desempenho dos sistemas de processamento automático de fala, nomeadamente os de reconhecimento de fala e os de síntese de voz; segundo, devido à convergência de dispositivos e à massiva produção de conteúdos multimídia, que passaram a requerer modos mais rápidos e eficientes de interação com os usuários (Salvador, et al., 2010).

Síntese de fala é o processo de criar uma réplica sintética de um sinal de fala assim como transmitir a mensagem a partir de uma máquina para uma pessoa (Tabet e Boughazi, 2011). Duas características são essenciais na classificação dos sistemas de síntese de fala: a qualidade do sinal de fala e o tamanho do vocabulário. Amostras de áudio pré-gravadas possuem qualidade real de sinal, contudo possuem vocabulário 
limitado, já os sistemas de Text-To-Speech (TTS) são capazes de gerar fala com boa qualidade com um vocabulário ilimitado.

O processo de síntese de um sistema de TTS é composto de duas fases principais. A primeira consiste no Processamento de Linguagem Natural (PLN), onde a mensagem de entrada é transcrita em uma representação de nível fonético e a segunda consiste na Geração da Fala em que as formas de onda de voz são geradas e a saída acústica é produzida. Essas duas fases são respectivamente chamadas de síntese de altonível e síntese de baixo-nível (Maia, 2006). Uma versão simplificada do processo é apresentada na Figura 2.

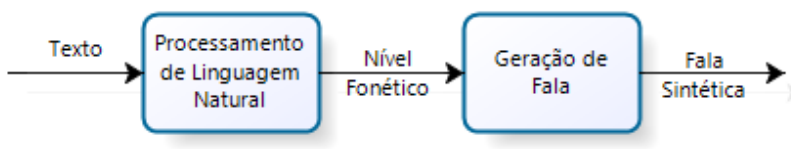

Figura 2. Descrição do processo de síntese de fala (Maia, 2006).

De acordo com Maia (2006) existem diferentes abordagens ou técnicas que podem ser utilizadas para geração de fala a partir das informações de uma locução. As mais utilizadas atualmente são: síntese de fala por seleção e concatenação de unidades; síntese de fala a partir de parâmetros gerados por Modelos Ocultos de Markov (no inglês: Hidden Markov Models - HMMs); e síntese por regras. Sintetizadores baseados em HMM têm sido muito apreciados recentemente por causa da possibilidade de obtenção de fala com boa qualidade a partir de uma pequena base de dados, e devido ao fato de que as características da voz podem ser facilmente modificadas.

Dois importantes recursos projetados para gerar fala usando HMM são HTS (HMM-based Speech Synthesis Toolkit) e MARY TTS (Modular Architecture for Research on speech sYnthesis). Em Maciel e Carvalho (2012) foi apresentado o treinamento de duas vozes por meio do HTS que geraram o motor VLSynthesizer, enquanto em Souza et al.(2014) foi apresentado o treinamento das mesmas vozes por meio do MARY TTS. Ambos os trabalhos foram incorporados ao Framework FIVE o que possibilita a utilização de vozes masculina e feminina em português falado no Brasil em múltiplas plataformas.

\section{Concepção e Desenvolvimento}

O processo de concepção do plugin Avatar Educação se baseou na literatura de Interação Humano-Computador (IHC), especificamente no método de Design da Interação proposto em por Preece et al. (2013), composto por quatro atividades: (1) identificação das necessidades do usuário; (2) desenvolvimento de alternativas de design; (3) construção de versões iterativas; e (4) avaliação do design.

A fase de identificação das necessidades do usuário envolveu uma pesquisa da situação atual das tecnologias disponíveis para identificar necessidades e oportunidades da ferramenta. Esta fase proporcionou o levantamento das principais notificações que deveriam ser apresentadas pelo assistente virtual (novos materiais postados, atividades pendentes, novas atividades postadas e mensagem do professor) que geraram como resultado as especificações dos requisitos funcionais, não funcionais e casos de uso. 
Na fase de desenvolvimento de alternativas de design foi utilizada a técnica de construção de cenários caricaturados (prototipagem) que serviu para criar uma situação de uso da ferramenta. Nessa fase foram realizados testes com usuários a fim de identificar possíveis problemas no fluxo de interação da interface, assim como possíveis problemas de usabilidade. As figuras 3.1 e 3.2 mostram os protótipos construídos.

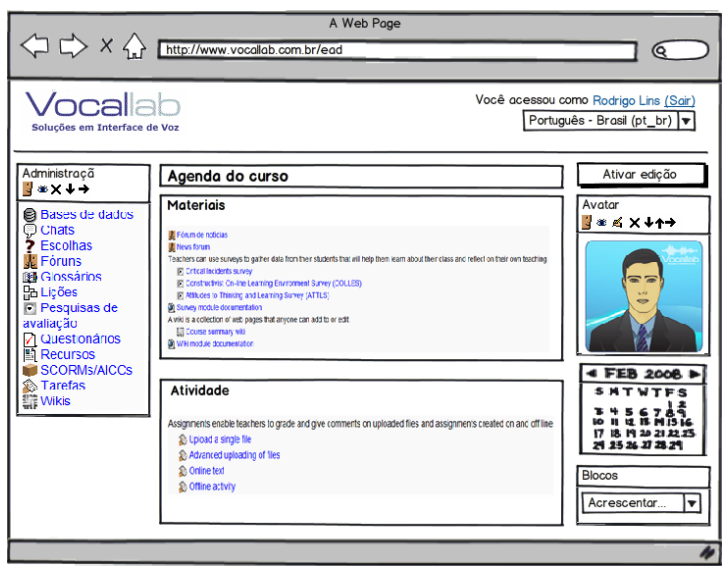

Figura 3.1 - Tela de apresentação

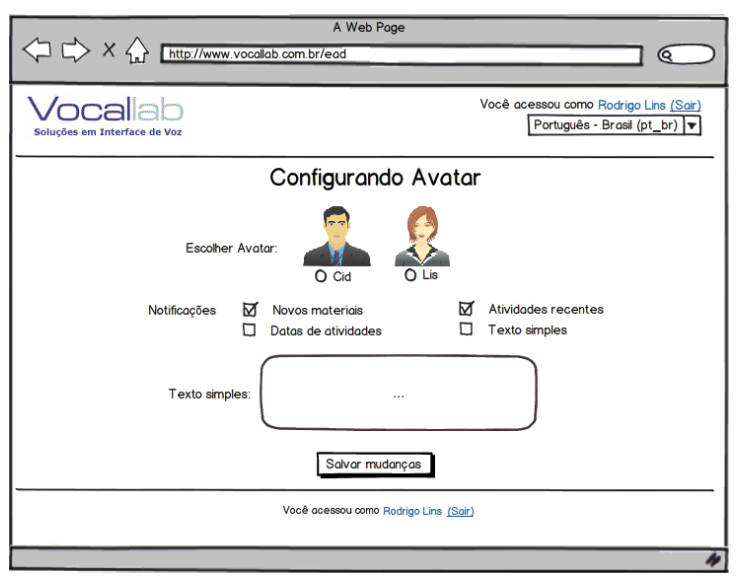

Figura 3.2 - Tela de configuração

A fase de construção da versão interativa iniciou por levantamento das principais tecnologias para o desenvolvimento do front-end e foi decidida a utilização da linguagem HTML5 associada ao CSS3. Os requisitos [instalação do bloco] e [configuração do bloco] seguiram o padrão do ambiente Moodle. Os requisitos [escolha do sexo] e [escolha do avatar] foram contemplados na área superior da tela de configuração e os requisitos [escolha das notificações] e [mensagem do professor] foram contemplados na área central da mesma tela. Para cada um desses requisitos foram implementadas as especificações existentes nos casos de uso específicos. As figuras 4.1 e 4.2 mostram as telas da versão interativa desenvolvidas.

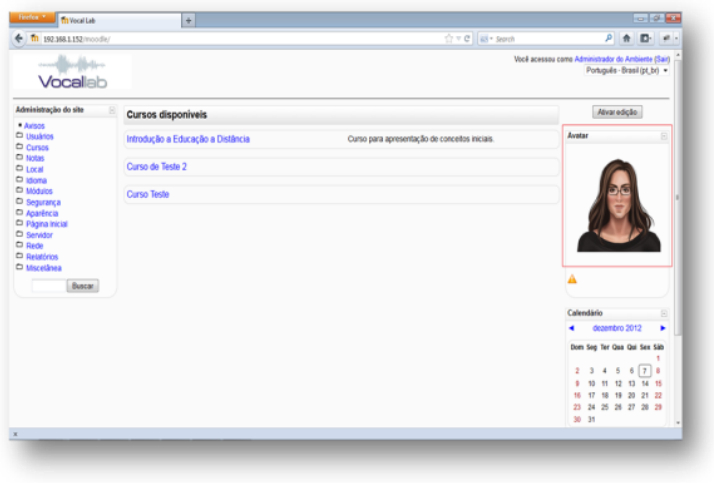

Figura 4.1 - Tela de apresentação

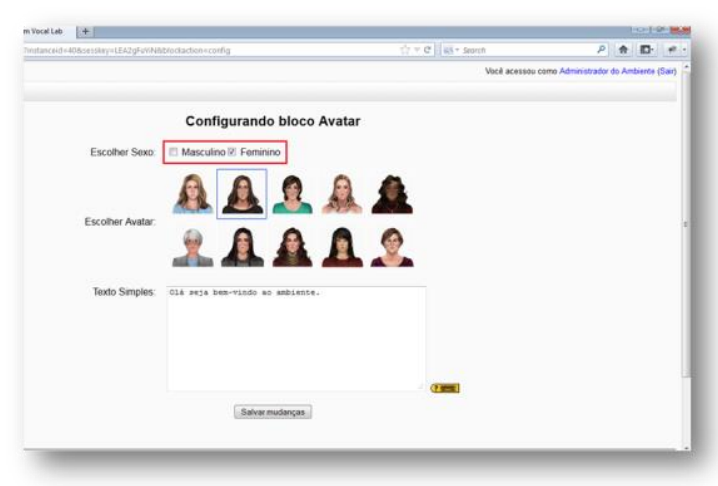

Figura 4.2 - Tela de configuração

Na fase de avaliação do design da versão interativa foi realizado um experimento com alunos de graduação em um ambiente real de ensino que detalhado na seção quatro.

\subsection{Arquitetura}

A plataforma Moodle é composta por uma estrutura cercada por plugins para prover funcionalidades específicas ao ambiente. Plugins são diretórios de códigos que podem ser simplesmente colocados dentro de qualquer instalação Moodle e ele será detectado, 
instalado e automaticamente estará disponível como ferramenta dentro da interface do Moodle.

Diversos tipos de plugins podem ser implementados na plataforma Moodle. Um dos mais populares são os blocos, que são pequenas caixas de informação ou ferramentas que podem ser movidas pela página. Fundamentalmente, um bloco consiste numa implementação de uma classe escrita em linguagem PHP que estende da classe block_base e como resultado final exibe um conteúdo HTML.

A arquitetura estabelecida para viabilizar o desenvolvimento do Avatar Educação utilizou o middleware Avatar Vocálico desenvolvido por Rodrigues et al. (2012) para a geração da sequência de visemas e do áudio sintético. Neste trabalho foi construído um bloco nos padrões do Moodle e um web service para que o plugin Avatar Educação enviasse as solicitações de texto e recebesse a sequência de visemas e o áudio sintético como retorno. A Figura 5 mostra uma visão geral da arquitetural desenvolvida.

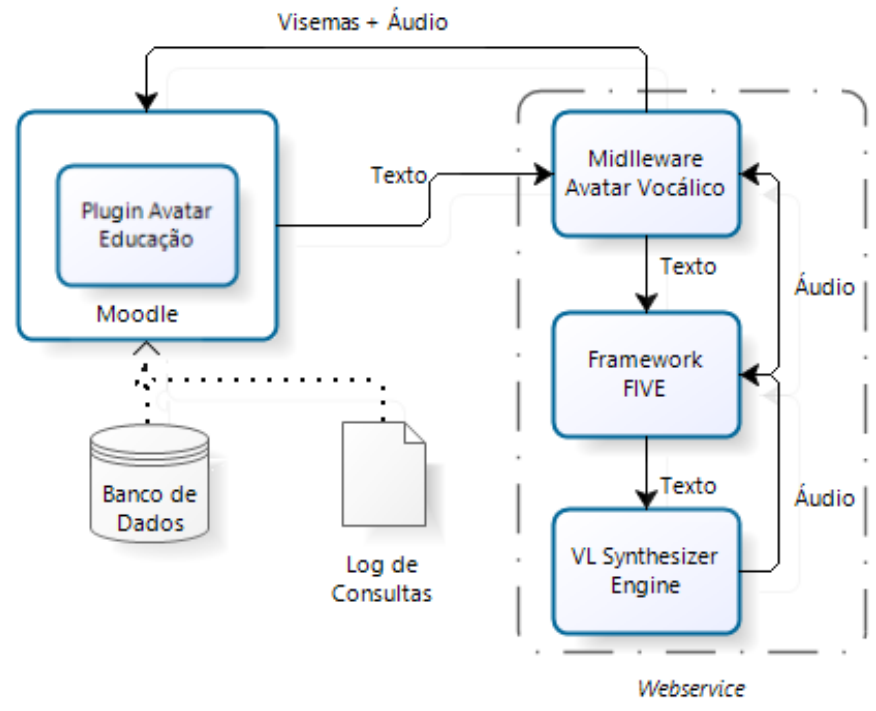

Figura 5. Arquitetura de integração do Plugin Avatar Educação

O processo de animação do Plugin Avatar Educação inicia a partir de uma consulta ao Banco de Dados do Moodle, conforme especificado nas opções de notificações escolhidas pelo usuário. O resultado dessa consulta é comparado com os logs armazenados num Log de Consultas. Caso o resultado da consulta esteja inalterado em relação à última consulta, não há necessidade de enviar uma solicitação via web service para o Avatar Vocálico. Caso o resultado da consulta retorne registros diferentes do armazenado no log, é envidada uma mensagem de texto para o Avatar Vocálico, que é responsável por gerar os visemas da animação e encaminhar para o FIVE uma solicitação de síntese de fala. O FIVE utiliza o motor VL Synthesizer para geração do áudio que é devolvido ao Avatar Vocálico. Os arquivos de visemas e de áudio ficam disponíveis no repositório do Avatar Vocálico, de onde são chamados pelo plugin para a geração da animação. 


\section{Experimentos Realizados}

Como forma de avaliar a eficácia da solução proposta, foi realizado um experimento quantitativo e qualitativo no qual foi possível fazer o uso do plugin em um ambiente real de ensino.

\subsection{Procedimentos}

A realização do experimento foi feita com 20 alunos da disciplina Introdução a Ensino a Distância do Curso de Sistemas de Informação da Universidade de Pernambuco. Esta disciplina foi ministrada de modo semipresencial, na qual foram disponibilizados materiais didáticos, solicitada a realização de atividades, e a disponibilidade de mensagens do professor através do avatar. Para a avaliação foram utilizados os $\log s$ para medir a frequência de uso da solução desenvolvida, e aplicado um questionário para avaliar a qualidade do assistente virtual por parte dos alunos.

\subsection{Resultados e Discussões}

Em relação aos resultados das análises dos logs de utilização da plataforma, durante o curso, tivemos valores de média e desvio padrão de 25,5 ( $\mathrm{SD}=27,08)$ utilizações do ambiente Moodle e uma média de 4,00 ( $\mathrm{SD}=5,39)$ postagens em fóruns de discussão. Outra variável coletada no estudo foi a quantidade de utilizações ao Avatar Educação. Em relação a esta variável tivemos uma média de 4,4 $(\mathrm{SD}=2,8)$ utilizações por aluno. Isto nos mostra que a variabilidade entre a utilização da aplicação foi relativamente alta.

Analisamos que, os acessos ao avatar ocorreram especialmente no início do curso, e que essa média de acessos se deu devido ao fato do aluno não ter tido a percepção visual, por meio da interface desenvolvida, de que havia novas mensagens disponíveis para ele. Este problema foi relatado ao final da disciplina pelos alunos e gerou um requisito de alerta visual para novas mensagens e notificações do avatar.

Em relação às variáveis coletadas através da aplicação de um questionário sobre a satisfação dos usuários, a Tabela 1 mostra os resultados obtidos.

\section{Tabela 1. Variáveis sobre qualidade da aplicação}

\begin{tabular}{|l|c|c|}
\hline \multicolumn{1}{|c|}{ Variável } & Média & Desvio Padrão \\
\hline Qualidade gráfica do personagem & 4,40 & 0,583 \\
\hline Qualidade da voz sintética & 3,85 & 0,654 \\
\hline Qualidade da animação & 4,35 & 0,726 \\
\hline Qualidade da sincronia labial & 4,15 & 0,654 \\
\hline
\end{tabular}

A variável de maior destaque foi em relação a qualidade gráfica do personagem. Esta variável obteve uma média de 4,40 $(\mathrm{SD}=0,583)$ dentre uma escala de variação de 1 (péssima) a 5 (ótima). A variável que obteve a menor média foi em relação a qualidade da voz sintética do personagem, esta variável obteve uma média 3,85 ( $\mathrm{SD}=0,654)$. Dentre as cinco variáveis sobre satisfação, podemos observar que todas tiveram valor médio acima do valor central da escala, demonstrando opiniões acima do valor Bom.

Em relação à análise qualitativa foi disponibilizado no questionário um espaço para respostas abertas, que puderam capturar a opinião dos alunos em relação a sugestões de melhorias. A ideia foi mapear as sugestões dos usuários a fim de refinar a 
versão atual do software bem como implementar novas funcionalidades em trabalhos futuros. A Tabela 2 mostra as principais sugestões levantadas pelos estudantes.

Tabela 2. Sugestões levantadas pelos estudantes

\begin{tabular}{|c|c|}
\hline [s1] & "Seria muito interessante ter um App que rode no Android com as notifica \\
\hline [s2] & "Sugiro que a velocidade da fala do avatar seja um pouco mais lenta". \\
\hline & "Em relação ao avatar, a voz poderia se aproximar mais da fala humana". \\
\hline & "O personagem poderia ser personalizável". \\
\hline
\end{tabular}

Em relação à sugestão [s1] pudemos pensar em como acoplar na arquitetura atual, um módulo que se comunique com aplicações móveis, utilizando as mesmas características gráficas da aplicação atual, isto facilitaria pois o aluno teria consigo a disponibilidade 24 horas do avatar lhe passando alertas e informações sobre $\mathrm{o}$ andamento da disciplina.

Em relação à sugestão [s2], esta é uma opção já disponível no motor VL Synthesizer. Cabe ao administrador do AVA solicitar a equipe técnica que ajuste os parâmetros de velocidade para tornar a voz mais agradável aos usuários. Em relação a sugestão [s3] este ainda é um ponto de várias pesquisas na área de interfaces de voz (Souza, 2014), a tentativa de aproximação da voz sintética a qualidade da voz humana é algo que tem evoluído.

Em relação à sugestão [s4], atualmente a solução desenvolvida conta com a possibilidade de escolha entre vinte personagens que podem ser escolhidos pelo professor. No entanto está implementada a funcionalidade para mudança de características do personagem. Este com certeza é um ponto importante pois a personalização de um assistente pode trazer uma maior familiaridade com os alunos.

Algumas limitações foram evidenciadas em relação a experimento. Uma delas foi o fato do mesmo ter sido realizado em uma turma de alunos da modalidade de ensino presencial. Neste tipo de modalidade, não é comum o uso intenso das ferramentas existentes no Moodle, tais como chat, atividades online, e postagens em fóruns, visto que maior parte das interações ocorrem em sala de aula. Desse modo, consideramos que devido a isto poucos dados foram coletados e a aplicação de análises inferenciais mais robustas ficaram limitadas, restando-nos apenas a possibilidade de análises descritivas.

\section{Conclusões e Trabalhos Futuros}

Este trabalho se propôs a apresentar o desenvolvimento de um assistente virtual integrado ao ambiente Moodle como forma de oferecer um suporte diário as atividades acadêmicas dos alunos do EAD. Em relação ao desenvolvimento consideramos que versão atual encontra-se estável e portável para qualquer ambiente Moodle. Em relação aos experimentos realizados pôde-se chegar a conclusão de que a solução desenvolvida atingiu os níveis de satisfação do usuário esperados. E, por fim, como trabalhos futuros, pretendemos realizar novos experimentos com turmas na modalidade totalmente a distância, capturando aspectos motivacionais e cognitivos sobre o uso do Plugin Avatar Educação, promovendo a geração de novos requisitos e o refinamento da solução. 


\section{Referências}

Costa, P. D. P. Animação facial 2D sincronizada com a fala baseada em imagens de visemas dependentes do contexto fonético. Dissertação de Mestrado, Universidade Estadual de Campinas, 2009.

Duarte, G. D., Costa, A. C. R. Uma Proposta para Modelagem de Personagens Virtuais Emotivos Utilizáveis em Ambientes de Educação a Distância. Anais do XXV Congresso da Sociedade Brasileira de Computação, 2005.

Gratch, J., et al. Creating interactive virtual humans: some assembly required. In IEEE Intelligent Systems, V.17, Issue 4, July, 2002.

Lester, J., Converse, S., Kahler, S., Barlow, T., Stone, B. e Bhogal, R. The Persona Effect: Affective Impact of Animated Pedagogical Agents. Anais da Conference on Human Factors in Computing Systems, 1997.

Lyra, F., Santos, N. Agentes de Software no Monitoramento de Alunos em Educação a Distância. Anais do Congresso Brasileiro de Informática na Educação, 2012.

Maia, R. S. Speech Synthesis and Phonetic Vocoding for Brazilian Portuguese based on Parameter Generation from Hidden Markov Models. Tese (Doutorado em Engenharia). Nagoya Institute of Technology, 2006.

Maciel, A., Carvalho, E. FIVE - Framework for an Integrated Voice Environment. Anais do International Conference on Systems, Signals and Image Processing, 2010.

Maciel, A., Carvalho, E. Integration and Evaluation of an HMM-Based Text-To-Speech System To FIVE. Anais do International Conference on Systems, Signals and Image Processing, 2012.

Preece, J., Rogers, Y., Sharp, H. Design de Interação - Além da Interação Homemcomputador, São Paulo, Bookman, 2013.

Prola, M. T. Modelagem de um Agente Pedagógico Animado para um Ambiente Colaborativo: Considerando Fatores Sociais relevantes. Dissertação de Mestrado, Universidade Federal do Rio Grande do Sul, 2003.

Rissoli, V. R. V. A., Santos, G. A. Monitoria Estudantil aliada a um Assistente Virtual Inteligente no Suporte à Aprendizagem via Internet, Anais do V Workshop de Arquiteturas Pedagógicas para Suporte à EAD mediada pela Internet, 2010.

Rodrigues, R. L., Maciel, A. M. A., Carvalho, E. C. B. Desenvolvimento de uma ferramenta para a produção de mídias utilizando personagem animado com síntese de voz, Anais do Congresso Brasileiro de Informática na Educação, 2012.

Salvador, V. F. M., Kawamoto, A. L. S., Oliveira Neto, J. S. An Empirical Approach for the Evaluation of Voice User Interfaces, Users Interfaces, v.1, pp 270, 2010.

Souza, D., Saturnino, L., Maciel, A. M. A. A Portability Evaluation of Brazilian Portuguese voices produced with MARY TTS. Anais do International Conference on Systems, Signals and Image Processing, 2014.

Tabet, Y., Boughazi, M. Speech Synthesis Techniques. A Survey. Anais do 7th International Workshop on Systems, Signal Processing and their Applications, 2011. 